$10 \mid 2018$

Devenir écrivain

\title{
Devenir Memmi
}

Fragments d'un journal intime inédit

Guy Dugas

\section{(2) OpenEdition}

12 Journals

Édition électronique

URL : https://journals.openedition.org/coma/1187

DOI : 10.4000/coma. 1187

ISSN : 2275-1742

\section{Éditeur}

Institut des textes \& manuscrits modernes (ITEM)

\section{Référence électronique}

Guy Dugas, «Devenir Memmi », Continents manuscrits [En ligne], 10 | 2018, mis en ligne le 15 mars 2018, consulté le 14 janvier 2023. URL : http://journals.openedition.org/coma/1187 ; DOI : https:// doi.org/10.4000/coma.1187

Ce document a été généré automatiquement le 14 janvier 2023.

\section{(c) $(1) \odot$}

Creative Commons - Attribution - Pas d'Utilisation Commerciale - Pas de Modification 4.0 International - CC BY-NC-ND 4.0

https://creativecommons.org/licenses/by-nc-nd/4.0/ 


\title{
Devenir Memmi
}

\author{
Fragments d'un journal intime inédit
}

\section{Guy Dugas}

\section{NOTE DE L'AUTEUR}

Printemps 1942. Dans la Tunisie en guerre, un jeune Juif de 22 ans désoeuvré, dont les conditions de vie sont plutôt problématiques, consigne dans son journal intime ses amitiés, ses amours, son besoin de culture et ses rêves d'aventure. Exprimant de manière récurrente un taraudant « besoin d'écrire ", il fait ses gammes dans une langue non maternelle qu'il apprivoise progressivement, non sans maladresses.

Ses lectures, à la fois philosophiques (Spinoza, Herbert Spencer) et littéraires (Kipling, Pirandello et surtout Gide, à qui il voue une grande admiration), expriment un balancement existentiel entre la connaissance et l'action. Quelques semaines plus tard, les aléas du moment, l'expérience des camps de travail obligatoire le pousseront à l'action, dont il témoignera par l'écriture - démarche qui annonce ses Portraits, analyse d'un vécu personnel.

Ses velléités d'écriture l'amènent également à égrener une chronique familiale qu'il intitule "Eux et moi". Déjà, il songe à puiser largement dans son journal pour composer « une espèce de roman autobiographique où je raconterai l'éloignement progressif d'un jeune homme de son milieu, de ses amis, pour rentrer en lui-même et à la découverte de la Connaissance ", ce qui sera, peu ou prou, le sujet de son premier roman, La Statue de sel, publié 10 ans plus tard. 


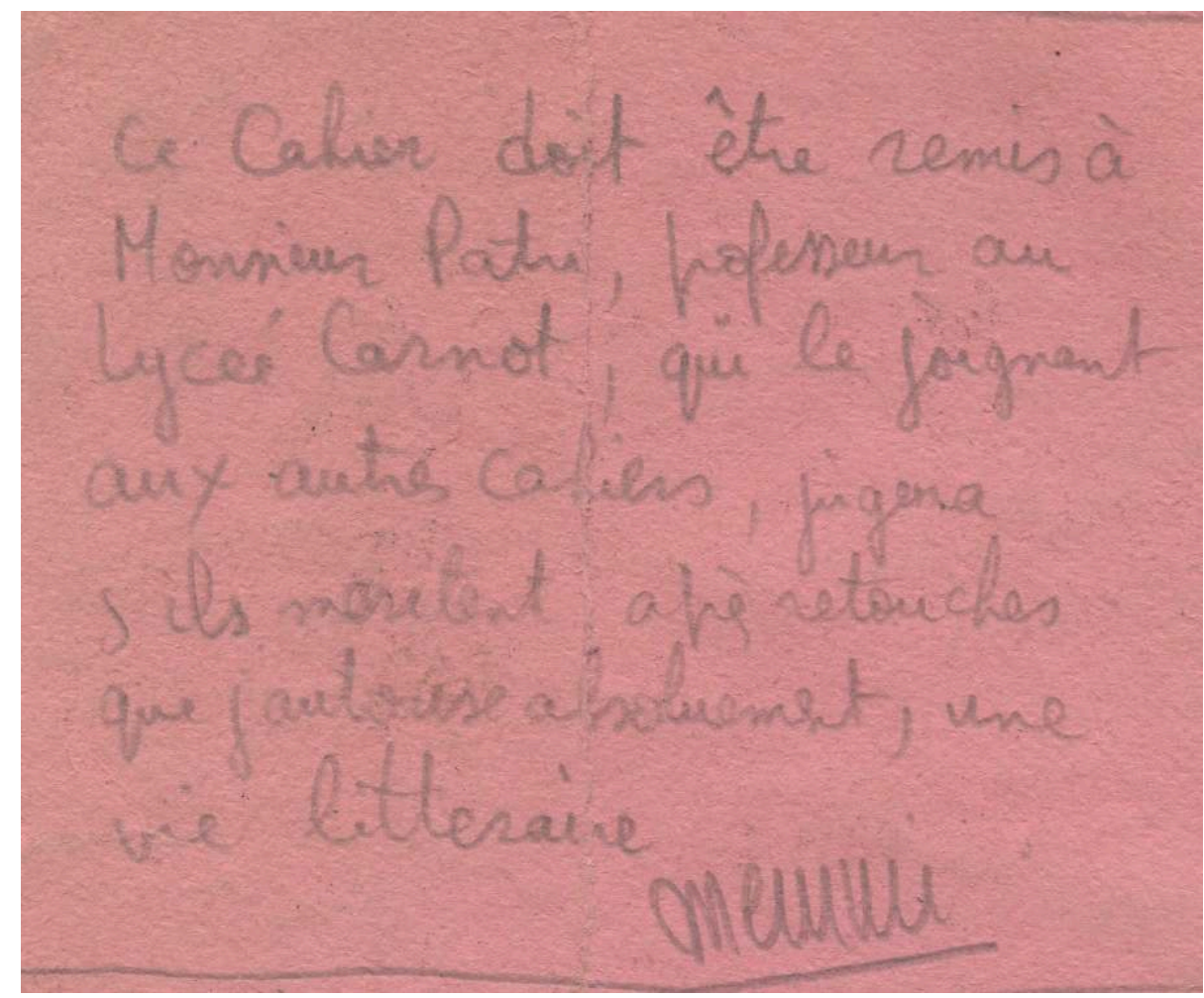

copyright : archives privées Albert Memmi

[ [Mars 1942] - J'ai besoin d'écrire. J'y pense tout le temps. Et je ne peux pas arrêter ma marche vers la connaissance, qui m'est aussi indispensable.

Je ne peux pratiquement concilier les deux. La discipline universitaire ne laisse pas beaucoup de temps. Et je veux réussir à mes examens.

2 J'ai Je cherche avec un besoin de plus en plus grand "le sujet", "mon œuvre".

Je suis sûr maintenant que je ne suis pas seulement un chercheur. La connaissance, aussi indipensable qu'elle est à ma vie, ne me comblera pas. Il me faut créer. Je suis un créateur. J'ai besoin de vivre ce que je pense.

3 Je suis sûr aussi que je n'ai aucune fidélité à mon oeuvre. Une fois que mon oeuvre est créée, c'est à dire une fois que je l'ai vécue, elle ne m'intéresse presque déjà plus.

Ce qui m'incline à penser que l'essentiel pour moi reste encore ma propre personne.

4 Je fréquente un peu Gide. Je l'ai surtout rencontré en ce point : que l'essentiel de mon oeuvre est et doit être mon propre Moi.

J'ai écrit "doit". Au fond, il s'agit peu de règles préétablies et d'impératifs. C'est un fait. On ne se sent pas ainsi, on l'est. D'ailleurs, les règles m'intéressent peu. 


\section{l'idée que jamais je n'atteindrai cette maîtrise. Je ne pense pas d'ailleurs qu'on puisse} dépasser cette habileté.

6 Mardi 24 mars 1942 - De même que le point de contact entre théorie et pratique (accord qui me paraît indispensable, qui ne l'est point pour certains, soit raisonnablement, ex. Patri, soit par inconséquence ou par lâcheté) me manque et cela m'exaspère. De même, le passage de mon cas individuel à la société, au cas de tous les autres hommes, je ne l'ai pas encore trouvé et cela me manque terriblement.

7 Samedi 28 mars 1942 - J'ai un besoin d'écrire qui me tenaille de plus en plus. Le besoin de connaître recule devant même. Je suis presque prêt à sacrifier 1 partie de la Connaissance pour créer.

8 Je compte écrire 1 espèce de roman autobiographique où je raconterai l'éloignement progressif d'un jeune homme de son milieu, de ses amis, pour rentrer en lui-même et à la découverte de la Connaissance. Large utilisation de mon journal.

9 Eux et moi - Je blaguais Bébé qui pose avec beaucoup d'assurance les questions les plus saugrenues. Mon père, mis en bonne humeur, prend le parti de Bébé et raconte quelques-unes de mes questions de gosse. Entre autres :

- Dis papa, qui c'est le plus fort, le forgeron ou l'agent de police?

L'histoire du chien qui me courrait [sic] derrière.

- Je veux courrir [sic], moi. Ça lui regarde !

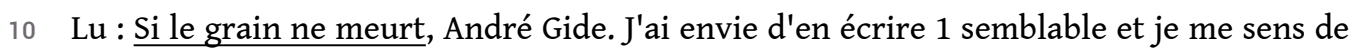
taille à le faire.

11 [1 feuillet demi-format dans le « garde-manger » de Memmi] Projets ${ }^{1}$

12 1) Si je suis reçu en faculté

a) piocher mes certificats restants.

b) remanier parallèlement mon dipôme et le présenter en $m[$ ême $\mathrm{t}[\mathrm{em}] \mathrm{ps}$.

13 2) Si je ne suis pas reçu ${ }^{2}$

a) travailler en vue de publication le diplôme.

b) commencer à réunir les éléments d'1 Histoire de la philosophie juive, future thèse de 
doctorat.

c) fonder une Revue d'études juives. Cette revue sera alimentée par les études particulières que je préparerai pour mon Histoire de [la philosophie] juive (d'une pierre deux coups).

d) parallèlement, pendant mes loisirs, écrire q[uel]que c[hose] d'artistique. Ex : Contes du folklore, etc.

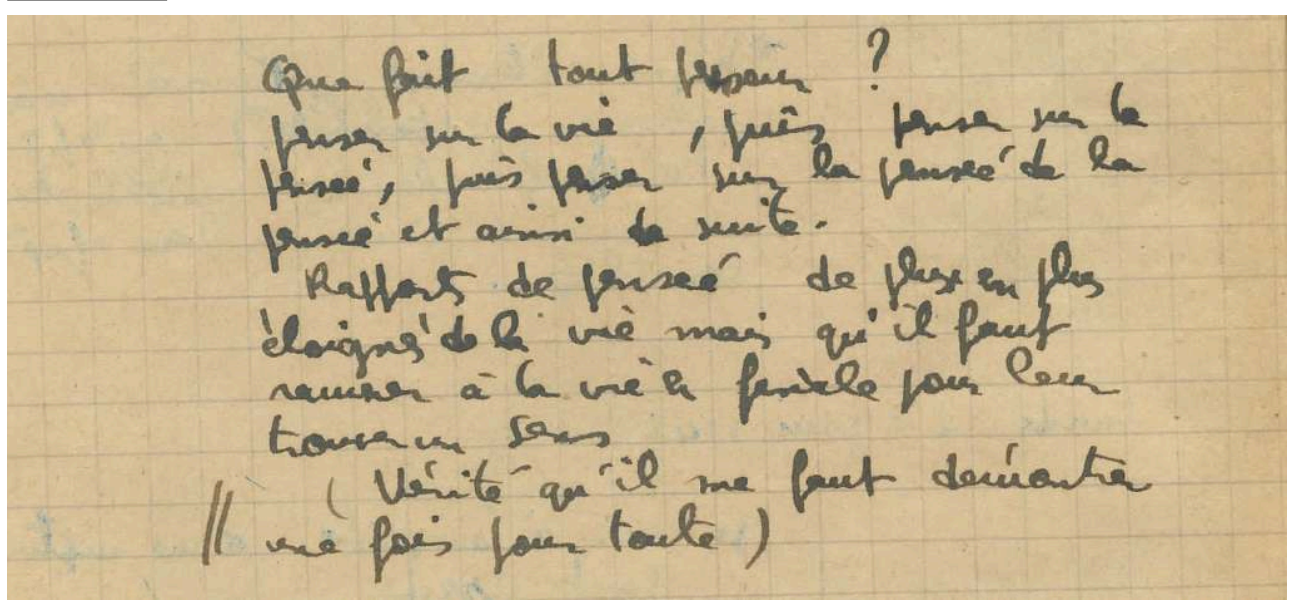

copyright : archives privées Albert Memmi

14 Jeudi 16 avril 1942 - Eux et moi

Denise qui tousse est obligée hésite à se faire poser des ventouses.

- Est-ce que ça fait mal ?

Je suis le seul à en avoir déjà essayé. Elle remue mes souvenirs. Alors Zozo, dédaigneux devant tant de pleutrerie et surtout sanctionnant là une méthode d'investigation fausse :

- Tu demande [sic] à Albert, mais peut-être que vous n'avez pas le même goût.

Les colères de Bébé sont terribles. Aussi tout le monde s'amuse à le mettre en colère, pour rire. En particulier Mamou. Alors Bébé, à bout, se sauve dans le couloir et hurle :

- C'est une maison de fou, on me rend fou, etc.

Samedi 25 avril - Lu Luigi Pirandello, Chacun son tour ${ }^{3}$. Ce n'est pas le meilleur Pirandello.

Je suis atteint d' 1 curieuse déformation. Chaque fois que je lis un livre, je ne le juge plus objectivement, mais par rapport à moi. Je me demande si je pouvais l'écrire, s'il me dépassait.

Lu Jean Martet, La Chasse à l'homme ${ }^{4}$. Un style habile, mais manque de génie. Tant pis.

Eux et moi - Bébé s'approche et me présente sa main, tous les doigts fermés, sauf l'index et le petit doigt.

- Qui tu veux? 
Ce qui dans notre langage commun signifie : si tu choisis le petit doigt, on est fâchés ; le grand, amis.

Devinant qu'il veut quelque chose, je le déçois intentionnellement en choisissant le petit. Tant pis.

Mais il n'a pas abandonné. Il revient cinq minutes après avec un nouveau plan de bataille. Il me présente le pouce et le majeur.

- Qu'est-ce que c'est?

Je ne comprends plus.

- Ah, je peux pas te dire. Choisis d'abord.

Je choisis le pouce. Il me dit en souriant :

- Alors, nous sommes amis.

C'était simple : il m'impose son amitié en m'ôtant la liberté de choix. Maintenant que nous sommes amis malgré moi, il demande :

- Tu m'aimes comment?

- Comme 1 kilo de cerises.

- C'est tout?

- Un kilo de cerises et un kilo de poires.

Nos comparaisons d'amitié sont très matérielles. Mais je suis loin de sa future demande. Il surenchérit pour me faire hausser.

- Moi, je t'aime comme la maison!

- Moi comme la table.

Il hausse encore pour m'obliger à monter malgré moi.

- Moi comme toute la rue!

- Moi comme la maison.

Ça va, il s'arrête. Je suis à la hauteur.

- Alors donne moi cinq sous.

Il les a gagnés. Je les lui donne.

Mercredi 27 mai 1942 - //Peut être joint à Eux et Moi//

Q[uan]d j'étais gosse, j'ai écrit un Ballet anonyme à une dame qui nous avait précéd succédé dans un appartement. Ele était fort prétentieuse et maniérée. Elle s'était répandue en propos aigres sur la tapisserie qu'elle avait été obligée de changer. J'ai éprouvé le besoin de la lui dire, mais j'ai été découvert. J'avais tourné autour de sa boîte aux lettres et j'avais été remarqué. Ce fut un beau scandale.

Déjà prophète.

Eux et Moi - Mamou barbouille au crayon la porte du cabinet : «Ida la folle,... » etc. Puis se dit qu'il sera battu. Alors il signe : «Albert». Albert, qui est grand, ne sera pas inquiété.

[Juin 1942] - La roue de l'aéromotor ${ }^{5}$ tourne lentement, uniformément. Je suis couché sur le dos. Au ciel, une multitude de nuages petits nuages blancs attend, immobile. Le vent se lève de temps en temps, et c'est alors un bruissement de tous les saccharomes. Ah, si j'avais une bonne langue, toute prête à servir, comme j'aurai [sic] exprimé toutes ces choses. Je sens l'inspiration, là, dans mon cœur, mais je ne sais pas dire grand- 
chose.

Quand j'en ai fini avec la terre, je me perds dans le ciel. Il est immense; les nombreux nuages argentés l'encombrent à peine. Aussi loin que va le regard, il ne découvre pas de limites. Toutes les hypothèses infinies sont justifiées. Il y a sûrement d'autres mondes et jamais une fin.

\section{NOTES}

1. Rajout au crayon rouge : " pour 1942 oct 1942 "

2. En marge de ce paragraphe, au crayon et écrit verticalement : «Et penser à gagner des sous ».

3. Luigi Pirandello (1867-1936), Il Turno, 1902. Trad française Yvonne Lenoir, éd. de la Nouvelle Revue critique, 1934.

4. Jean Martet (1886-1940), La Chasse à l'homme. Paris, Albin Michel, 1941.

5. Instrument à hélice mû par le vent permettant de puiser l'eau dans les puits.

\section{INDEX}

Mots-clés : Albert Memmi, Tunisie, littérature maghrébine de langue française, journal intime

\section{AUTEUR}

\section{GUY DUGAS}

Chercheur associé à l'équipe Manuscrits francophones, ITEM (CNRS-ENS) 